\title{
Origen de las pymes en Colombia: ¿consecuencia de una acertada política nacional de emprendimiento o fruto de la ausencia de oportunidades laborales para los colombianos?
}

\author{
págs. 217-225
}

\author{
Grupo de investigación: Centro de Investigación y Desarrollo Empresarial CINDE \\ Línea de Investigación: emprendimiento \\ Martín Felipe Cuevas Oviedo •
}

Recibido: 13 de junio 2013

\section{RESUMEN}

Este artículo pretende identificar la verdadera razón por la cual un emprendedor colombiano decide abrir una empresa en Colombia. Si bien el Gobierno Nacional a partir del año 2009, presentó un documento con la Política Nacional de Emprendimiento, desafortunadamente Colombia ha sido históricamente un país que se caracteriza por la informalidad en el mercado laboral. Por lo tanto, se presenta un análisis sobre el rol del Estado frente a la Política Nacional de Emprendimiento, así como los cuatro indicadores de medición mundial que el Gobierno Colombiano debe considerar, para que la política de emprendimiento nacional tenga una correcta medición.

También se toma en consideración las principales motivaciones que tiene un emprendedor colombiano para la apertura de una empresa, así como la presentación de datos contundentes, sobre la discriminación sistemática que tiene el sexo femenino en el mercado laboral colombiano. Se destacan algunos resultados obtenidos en un estudio del Global Entrepreneurship Monitor - GEM sobre el emprendimiento en Colombia. Finalmente, se realiza un breve repaso sobre el marco jurídico del emprendimiento a nivel nacional.

Palabras Clave: Emprendimiento, informalidad, Pymes, política nacional, mercado laboral.

Aceptado: 16 de octubre de 2013

\section{ABSTRACT}

This article pretends to identify the real reason why a Colombian entrepreneur decides to start a business in Colombia. While the National Government since the year 2009, presented a document called the National Policy on Entrepreneurship, unfortunately Colombia has historically been a country characterized by informality in the labor market. Therefore, it presents an analysis of the role of the State about the National Policy Entrepreneurship and the four global indicators measuring the Colombian Government should consider, for national entrepreneurship policy has the correct measurement. It also take into account the main motivations that have a Colombian entrepreneur for starting a business, as well as the presentation of convincing data on the systematic discrimination that has the female at the Colombian labor market. Finally, it presents some results obtained in a study by the Global Entrepreneurship Monitor - GEM on entrepreneurship in Colombia. Finally, it shows a brief review of the legal framework of entrepreneurship nationwide.

Keywords: Entrepreneurship, informality, SMEs, national policy, labor market.

- Docente Investigador Universidad de América, Programa de Ingeniería Industrial. 


\section{INTRODUCCIÓN}

Durante el año 2011, las medianas empresas en el país tuvieron un crecimiento superior al $15 \%$ con respecto al año 2010 y las ventas superaron los $\$ 180$ billones de pesos, de acuerdo al reporte generado por la Superintendencia de Sociedades. También vale la pena destacar que durante este mismo año las pequeñas empresas en Colombia lograron superar los $\$ 100$ billones en ventas. Si uno compara estas cifras con las ventas durante el mismo periodo de las grandes empresas, ese reporte afirma que estuvieron por el orden de los $\$ 188$ billones.

Todo lo anterior nos lleva a concluir que Colombia es un país promotor de Pymes, y que el darles una real dimensión e importancia dentro de la economía nacional, podría beneficiar en gran medida el crecimiento y prosperidad económica, especialmente en estas épocas de crisis a nivel mundial, donde el famoso "efecto domino", está acabando lentamente con diferentes economías a nivel internacional. Está más que demostrado que cuando un país tiene una política adecuada de emprendimiento, puede minimizar estos impactos económicos globales a través de la generación de empleo por medio de las pymes.

Se dice que en la actualidad existe una cifra cercana a las 23.000 pymes en Colombia, que generan el $76 \%$ del empleo nacional, donde el $56 \%$ de ellas se dedican al sector servicios y el $19 \%$ a la industria (Revista Dinero, Artículo "Pyme: Sinónimo de oportunidad y desafío, 2012). Sin embargo, se estima que la rentabilidad sobre el patrimonio actual para las grandes es de casi $9 \%$, para las medianas la cifra es de $8,4 \%$ y para las pequeñas es apenas de $5,1 \%$, de acuerdo a datos suministrados por la Superintendencia de Sociedades.

Por lo tanto, estas cifras demuestran que a pesar que Colombia cuenta desde el año 2009 con una "Política Nacional de Emprendimiento" presentada por el Gobierno Nacional, algo está sucediendo en su implementación, ya que el ci- clo de vida de las Pymes es bastante corto y su rentabilidad con respecto a las grandes empresas no es la mejor, donde estas últimas se lleven la mejor tajada del pastel.

En consecuencia, se debe realizar un minucioso análisis sobre la "Política Nacional de Emprendimiento" adoptada en Colombia desde el 2009, verificando su real implementación, sus resultados y si verdaderamente han logrado potencializar el sector de las Pymes en el país.

Desafortunadamente, de acuerdo al ranking del año 2011 respecto al coeficiente de Gini (Programa de las Naciones Unidas para el Desarrollo (PNUD), "Informe sobre Desarrollo Humano 2011 - Sostenibilidad y equidad: Un mejor futuro para todos", 2011), indicador económico que mide el nivel de distribución de ingresos dentro de la economía de un país, Colombia ocupa el penoso lugar 150, de un total de 160 países medidos a nivel mundial. Esto quiere decir, que Colombia es uno de los países más desiguales en cuanto a la distribución de riqueza a nivel mundial.

Al existir tales desigualdades en Colombia, se podría generar la hipótesis que el emprendimiento en Colombia y la creación de empresa, es consecuencia en gran medida por la inequidad social y por lo tanto, por la falta de oportunidades laborales para el grueso de la población. Se podría afirmar también, que de ser cierta ésta premisa, Colombia estaría recorriendo el camino errado para la creación de empresa, ya que el emprendimiento y la creación de empresa debe ser fruto de una política nacional coherente frente al emprendimiento y la innovación, y no como consecuencia de desigualdades sociales y económicas.

Estos malos resultados para Colombia pueden generar una gran oportunidad para las Pymes, que siendo las mayores generadoras de empleo en Colombia, podrían contribuir simultáneamente a su progreso económico y a la expansión económica del país, reduciendo el desempleo y mejorando la distribución de la riqueza. 


\section{MÉTODO}

\subsection{Política nacional de emprendimiento de Colombia}

De acuerdo al documento "Política Nacional de Emprendimiento de Colombia", aprobado por el Gobierno Nacional en el mes de julio del año 2009, por medio del Ministerio de Comercio, Industria y Turismo (Ministerio de Comercio, Industria y Turismo, 2013), se elaboró un marco conceptual para que el país tuviera clara una política de creación de empresa, emprendimiento e innovación.

Como primera medida se hace referencia al marco normativo (Política Nacional de Emprendimiento, Ministerio de Comercio, Industria y Turismo, Julio de 2009), donde se presentan los antecedentes jurídicos que tienen que ver con las leyes y decretos que se han expedido con anterioridad sobre éste tema. También se hace referencia a los documentos CONPES que se han expedido en el pasado, donde se destaca el CONPES 3527 del 23 de junio de 2008, sobre la Política Nacional de Competitividad y Productividad.

De acuerdo a este documento, el Estado tiene tres roles principales respecto al fomento del emprendimiento en Colombia (Política Nacional de Emprendimiento, Ministerio de Comercio, Industria y Turismo, Julio de 2009):

- Promotor de la alianza público - privada académica.

- Facilitador de las condiciones para el emprendimiento.

- Desarrollador de la dimensión local, regional, nacional e internacional del emprendimiento.

Así mismo, el Gobierno considera que para poder realizar una correcta medición sobre los diferentes aspectos que conforman la política de emprendimiento, se deben considerar cuatro indicadores de medición mundial (Política Nacio- nal de Emprendimiento, Ministerio de Comercio, Industria y Turismo, Julio de 2009):

- El Global Entrepreneurship Monitor, que mide la actividad empresarial en 42 economías.

- El Índice Doing Business, que proporciona una medición objetiva de las regulaciones para hacer negocios y su aplicación en 181 países.

- El Scorecard de LAVCA (Latin American Venture Capital Association), que mide el ambiente para que los fondos de capital privado (equity) y de riesgo (Venture), inviertan en países de Latinoamérica y del Caribe, comparando 12 economías de la región, e incorpora como uno de sus componentes el emprendimiento.

- Cifras del Sistema Nacional de Incubación.

\subsection{Motivaciones para creación de empresas por género en Colombia}

De acuerdo a la encuesta realizada por el Global Entrepreneurship Monitor (GEM) a la población adulta en Colombia durante el año 2007, respecto a la motivación para la creación de empresa, claramente existe una diferencia entre ambos sexos respecto a los resultados obtenidos.

Mientras que para las mujeres el factor motivacional que prevalece es la necesidad (49\%), aunque la oportunidad tuvo un resultado significativo (45\%), para los hombres la principal motivación es la oportunidad (61\%), mientras la necesidad (36\%) sigue teniendo también una cuota interesante. Este resultado se puede deber en gran medida a la inequidad de oportunidades laborales que tiene la mujer en Colombia con respecto al hombre.

Claramente lo que se puede observar en Colombia normalmente es que la mujer en promedio devenga menos que el hombre en la mayoría de profesiones, lo cual puede ser una conse- 
cuencia para que la necesidad sea su principal motivación para la creación de empresa. Todo lo contrario para el sexo masculino, donde prima el factor de oportunidad en el momento de iniciar un nuevo negocio. Todo esto nos lleva a concluir que claramente, Colombia es un país donde se presenta una discriminación laboral contra el sexo femenino, lo que puede estar generando problemas en el tema de emprendimiento, debido a que la mujer colombiana está creando empresa por falta de oportunidad laboral, y no por un incentivo que le pueda presentar su entorno económico y laboral, ni por políticas tomadas por el Gobierno Nacional.

\section{Gráfica No. 1}

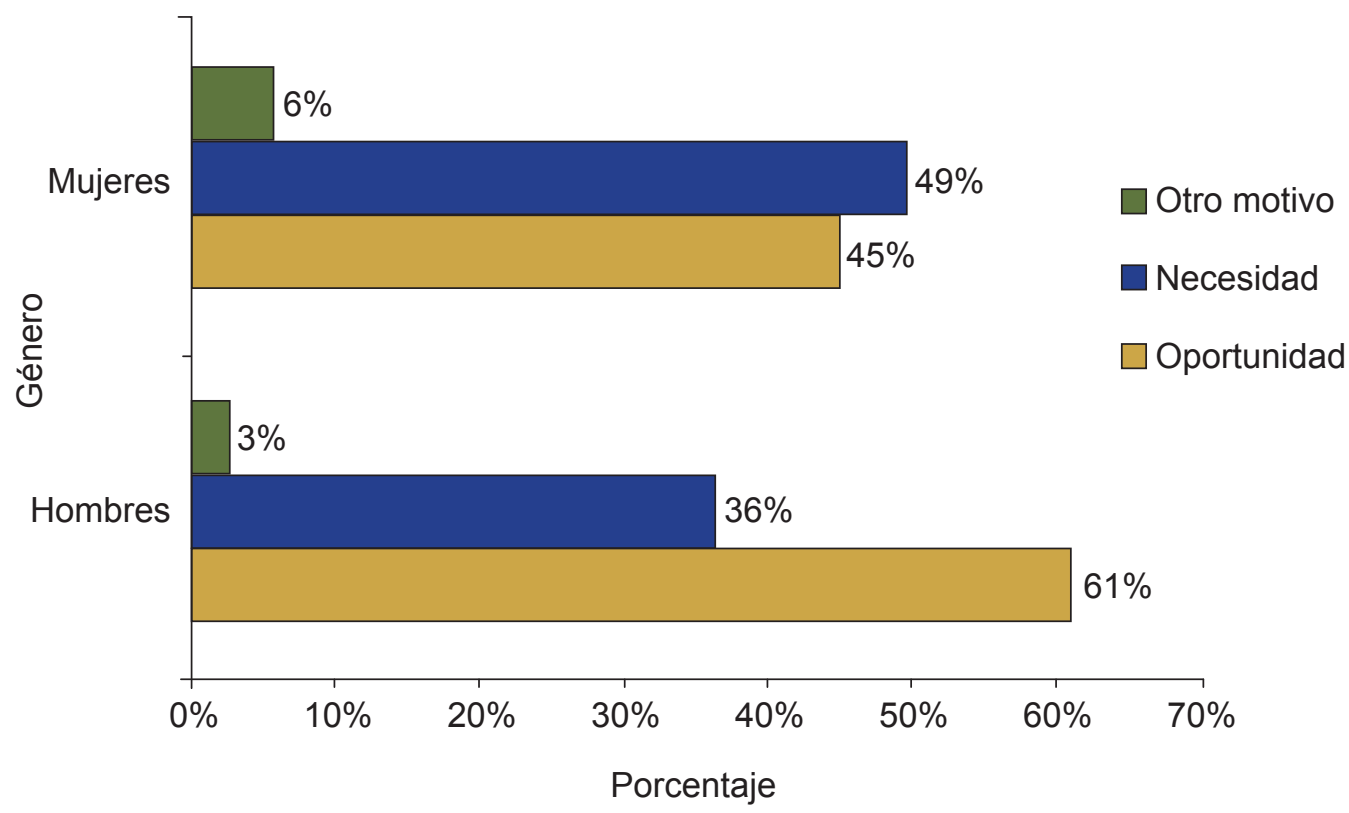

Fuente: encuesta a la población adula GEM 2007

\subsection{Discriminación laboral de la mujer en Colombia}

De acuerdo al artículo "La discriminación laboral tiene cara de mujer" elaborado por Lorena Álvarez Ossa, del portal razónpublica.com, se pueden obtener los siguientes datos escalofriantes respecto a la situación laboral actual de la mujer en Colombia (Portal razonpublica.com, artículo "La discriminación laboral tiene cara de mujer", Lorena Álvarez Ossa, 2010)

- Según estadísticas del DANE, 2008, Colombia tiene 17.259.000 mujeres en edad de trabajar. De ellas, el $46,3 \%$ hace parte de la población económicamente activa (PEA) y un $53,7 \%$ se considera población inactiva.
- Las mujeres que hacen parte de la PEAy están ocupadas, son aproximadamente 6.866.000. De ellas 3.887 .000 están en las 13 áreas metropolitanas, donde más de la mitad se ubica en la economía informal, en su mayoría en empleos precarios y de poco amparo en los derechos establecidos por la ley.

- Las mujeres que hacen parte de la PEAy están inactivas, suman 9.263.000. Un $62 \%$ de ellas están dedicadas al trabajo reproductivo, es decir al cuidado de la vida en el hogar y el mantenimiento de la fuerza de trabajo; mujeres que históricamente han sido invisibles a los análisis económicos, ya que su labor socialmente no se considera trabajo. 
- En las 13 áreas metropolitanas el 59,2\% de las mujeres ocupadas (unas 2.302.000) está en la economía informal.

- En las 13 áreas metropolitanas del país, en 2008 hubo una redistribución en el empleo femenino del sector formal de la economía, según posición ocupacional.

- En el último año (relación 2007-2008), considerando la totalidad de la población económicamente activa, en el país hubo 67.000 puestos de trabajo menos para las mujeres y 185.000 más para los hombres. Quiere decir que la población femenina sigue teniendo menores oportunidades de acceso al mercado laboral, lo que se traduce en pérdida del bienestar y endurecimiento de las desigualdades entre éstas y los hombres.

- Sin embargo, hay obstáculos en el mercado laboral que impiden un mayor acceso y movilidad de las mujeres en el mundo del trabajo productivo. Esto está relacionado con el hecho de que en la economía formal a las mujeres se les exigen mayores niveles de preparación (un año en promedio) que los exigidos a los hombres.

- En las 13 áreas metropolitanas consultadas por el DANE, en el 2007 las mujeres recibían, en promedio, el $74,31 \%$ de los ingresos que percibían los hombres. Fue más alto este promedio en el sector formal de la economía: el $83,58 \%$ de lo que recibían los hombres. Porque en el sector informal a las mujeres les va peor: el promedio de sus ingresos sólo llegaba al $65,40 \%$ de los hombres ocupados en este mismo sector de la economía.

- De las mujeres ocupadas en 2008, 3.199.000 están en condiciones de subempleo. Quiere decir que de cada 100 que están trabajando, 40 tienen empleos inadecuados, ya sea por tener mayores competencias para desarrollar la labor que desempeñan; o porque se ocupan menos de 48 horas; o reciben menos ingresos.
- Los datos del DANE señalan un aumento del desempleo de las mujeres. De las 7.996.000 que en el 2008 hacían parte de la población económicamente activa, 1.130 .000 mujeres (el 14,1\%) estaban desempleadas. Ese año, con respecto a 2007, se registraron 35.000 empleos menos para las mujeres.

- En contraste, en 2008 se crearon 185.000 empleos más para los hombres. La tasa de desempleo masculina este año se mantuvo sin variación con relación al $2007: 8,4 \%$, seis puntos inferior a la tasa de desocupación de las mujeres.

Los datos anteriores presentan la situación precaria en que se encuentran las mujeres a nivel laboral, y como el género determina de manera significativa la posibilidad de creación de empresa en Colombia. Este es un tema a revisar, teniendo en cuenta que históricamente la mujer tiene menos oportunidades laborales y menores ingresos salariales en nuestro país, lo que la hace un segmento vulnerable y bastante apto para la creación empresa.

\subsection{Principales hallazgos del GEM Colombia 2007}

De acuerdo al estudio realizado por el Global Entrepreneurship Monitor - GEM durante el año 2007 en Colombia, se pudieron identificar algunos factores claves que se presentan a continuación (Política Nacional de Emprendimiento, Ministerio de Comercio, Industria y Turismo, Julio de 2009):

- Colombia ocupa el tercer lugar en el mundo en la Tasa de Actividad Empresarial - TEA de un total de 42 países. La TEA del país está alrededor del $23 \%$.

- Cerca de 6 millones de adultos colombianos, entre 18 y 64 años, están asumiendo el reto de crear nuevas empresas.

- El $14 \%$ de las iniciativas inician su actividad empresarial en el marco de la formalidad. 
- En Colombia las fuentes informales financian la creación de empresas.

- El 93,3\% de los inversionistas manifestó que sus inversiones se hacen en empresas creadas por familiares cercanos, amigos, vecinos o colegas.

De lo anterior se deduce que definitivamente Colombia es un país emprendedor por naturaleza, donde generalmente aparece bien ubicado en los diferentes rankings que se realizan tanto a nivel regional como mundial. Este emprendimiento se refleja en la actualidad, no solamente en la población adulta, sino en la población juvenil, la cual, cada vez presenta menor temor respecto a la actividad empresarial. En este punto es clave el tema de la financiación de proyectos que le permita a cualquier persona independientemente del extracto social de donde provenga, el poder tener la misma oportunidad para la creación de empresa.

Sin embargo, el tema de la informalidad sigue prevaleciendo en los creadores de empresa, el cual, es un tema identificado con claridad por el Gobierno Nacional, quien se encuentra generando medidas de choque para enfrentarlo. También sorprende que la financiación para la creación de empresas provenga en su mayoría de fuentes informales. Esto demuestra que tanto el sector público como el sector privado en Colombia deben involucrarse más en este tema y aumentar su participación en la financiación de este tipo de proyectos.

Así mismo, la encuesta realizada por el Global Entrepreneurship Monitor (GEM) a la población adulta en Colombia durante el año 2007, pudo establecer los sectores preferidos por los emprendedores colombianos para la apertura de un negocio. Como se puede apreciar en la gráfica No. 2, en Colombia el sector de servicios de consumo (61\%) es de lejos el sector de mayor incursión para la creación de nuevas empresas. Sin embargo, la cifra preocupante es la disminución del sector de transformación (26\%) con respecto a los años anteriores, teniendo en cuenta que es éste sector el responsable del desarrollo de nuevas industrias que claramente afectará las variables de innovación y tecnología en el país. Por lo tanto, el Gobierno debe hacer énfasis y enfocar esfuerzos en reactivar el sector dela transformación por el bien de la industria nacional.

\section{Gráfica No. 2}

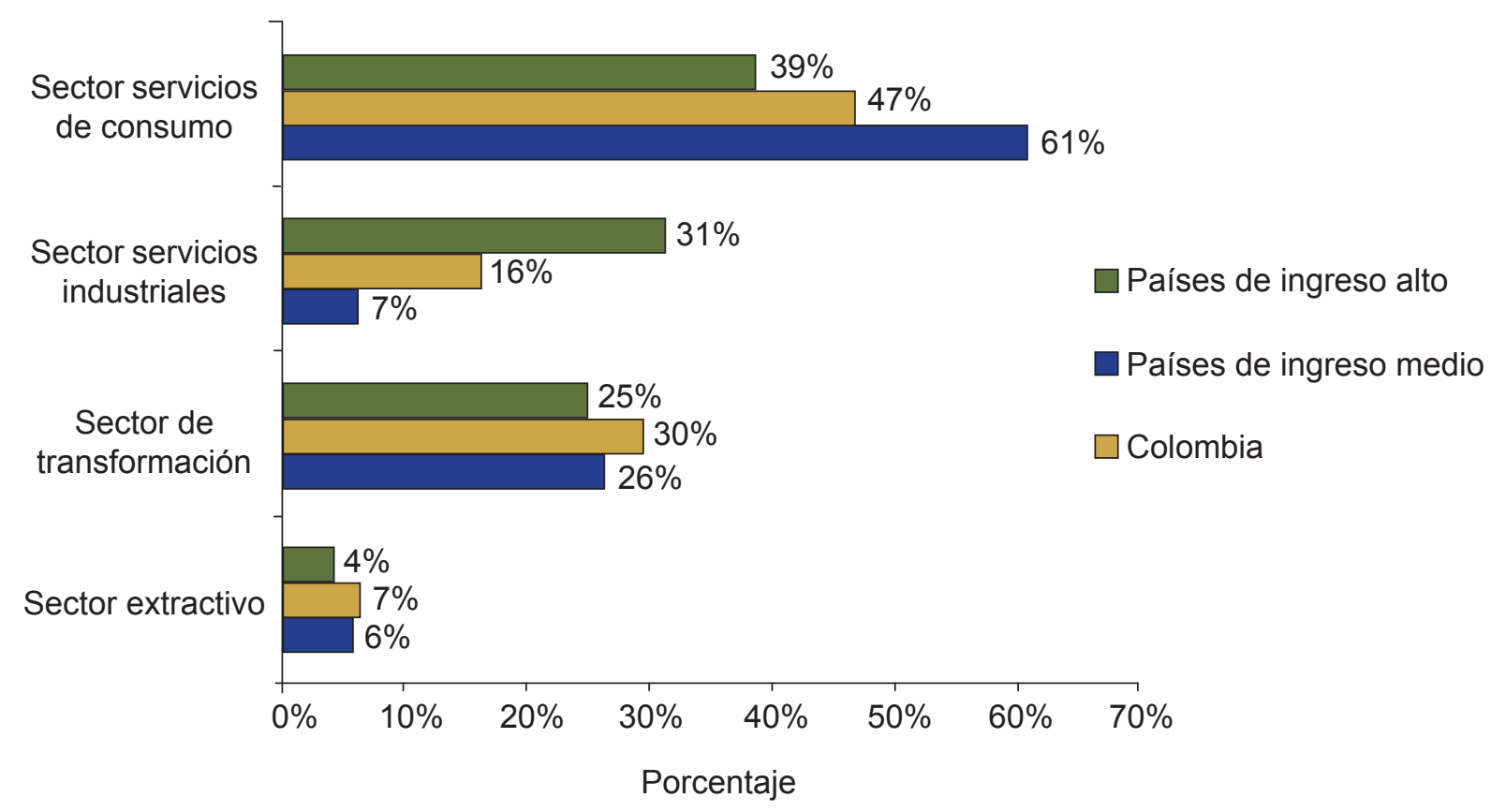

Fuente: encuesta a la población adulta GEM 2007 


\subsection{La informalidad laboral en Colombia}

De acuerdo al Diario Portafolio (Diario Portafolio, Sección Economía, Artículo "Informalidad laboral en Colombia llega al 51,3\%", 2012), la informalidad en Colombia durante el año 2012, alcanzó la cifra del $51,3 \%$. Este dato se puede considerar bastante preocupante, puesto que de acuerdo al informe del Banco de la República (Banco de la República, Informalidad laboral en las áreas urbanas de Colombia, Luis Armando Galvis, 2012), respecto al tema de parafiscales, por la falta de aportes a seguridad social, cerca de seis de cada diez empleados se ubicaría en el sector informal. Por lo tanto, el perfil de los informales en Colombia se caracteriza por presentar bajos niveles educativos, menores niveles de ingreso y se desempeñan en establecimientos de menor tamaño que los trabajadores formales.

A nivel regional, los niveles más altos en el grado de informalidad lo presentan ciudades que están por fuera del centro de actividad económica del país, especialmente las ciudades que no se encuentran en el trapecio conformado por Bogotá, Cali, Medellín y Bucaramanga. Se puede afirmar que para las 23 ciudades principales del país, la tasa de informalidad aproximada es del $42 \%$ respecto a las personas que no tienen "un contrato de trabajo". Esta cifra lo que demuestra es la baja productividad e innovación, así como la baja recaudación de impuestos en el país.

Otro aspecto clave que se deriva de la informalidad, es la movilidad del capital humano entre el sector "secundario" y las respectivas barreras para ingresar al sector "moderno". Esto está ligado directamente con la falta de calificación requerida por las personas para optar a un "trabajo formal", lo que conlleva a que se origine un desbalance entre la demanda y la oferta del mercado laboral.

En países subdesarrollados como el nuestro normalmente prima el trabajo no calificado y la poca capacitación técnica y profesional de las personas, lo que sumado a las prácticas monopólicas y oligopólicas de algunos sectores, genera un caldo de cultivo para la informalidad en Colombia. También existe el caso del personal calificado que es expulsado del sector formal y que por lo tanto para poder subsistir debe acudir al mercado informal.

En la tabla No. 1 se pueden apreciar los principales factores que explican en gran medida, el desbalance que se produce entre la oferta y la demanda en el mercado laboral.

Tabla No. 1

\begin{tabular}{|c|c|}
\hline & Oferta \\
\hline $\begin{array}{l}\text { Escaso desarrollo estructural de la economía (baja } \\
\text { diversificación económica) }\end{array}$ & $\begin{array}{l}\text { - La transición demográfica en su segunda etapa, } \\
\text { cuando ya ha disminuido la tasa de mortalidad } \\
\text { pero la tasa de natalidad es aún alta }\end{array}$ \\
\hline - Uso de tec & \\
\hline - Bajos niveles de inversión & \\
\hline $\begin{array}{l}\text { La política de disminución del tamaño del Estado, } \\
\text { cuyos trabajadores por definición son formales }\end{array}$ & $\begin{array}{l}\text { - La mayor participación de los miembros familia- } \\
\text { res diferentes al jefe del hogar, especialmente } \\
\text { mujeres }\end{array}$ \\
\hline
\end{tabular}

Fuente: elaboración propia con base en Uribe y Ortiz (2006). Para la oferta los autores citan a Harris y Todaro, 1970. 
A lo largo de la historia humana se han generado posturas encontradas entre la definición del perfil de un trabajador informal. Para algunos, un trabajador informal se caracteriza por ser una persona emprendedora e informal por voluntad propia. Para otros, un trabajador informal es una persona pobre y que son informales por necesidad. Sin embargo, una de las principales conclusiones a las que se puede llegar frente al tema de la informalidad en Colombia, es que definitivamente los trabajadores informales presentan una situación más precaria respecto a su nivel de ingresos, en comparación con un trabajador formal. Por lo tanto, son personas de bajos ingresos, que habitan las ciudades menos prósperas del país, con un bajo nivel educativo y que en su mayoría pertenecen al sector femenino y de los jóvenes.

\subsection{Marco jurídico para la creación de empresa en Colombia:}

De acuerdo a la Coordinadora de Investigación de UNISINU, Carmen Ortega Otero, (Revista de Investigación Semillas, 2008), aunque en Colombia existen normas y leyes para promover la apertura de empresas, estas no son claras y en algunos casos se pueden considerar bastante cuestionables. El mayor problema que enfrentan estas leyes, es que involucran organismos que no están articulados adecuadamente y por lo tanto no son normas fáciles de aplicar para un emprendedor. $Y$ en algunas ocasiones presentan un carácter ambiguo.

Las formas de financiación tampoco son claras y en general las leyes promueven el fortalecimiento de las empresas y no la creación de éstas, que debería ser su principal objetivo. Existe una tramitología que es perjudicial para el fomento de la nueva empresa, puesto que en la actualidad se deben llevar a acabo alrededor de 18 a 20 trámites para poder abrir un nuevo negocio, que varía en un periodo de 55 a 60 días, sumado a los costos que implica para el emprendedor. Por lo tanto, se debería realizar una especie de reingeniería en la parte legal de la apertura de empresas en Colombia, haciendo hincapié en incentivos económicos que provengan de fondos de capital de riesgo privados.

\section{CONCLUSIONES}

Cuando uno se remite a la mayoría de los rankings sobre emprendimiento y apertura de empresa en la región, queda claro que Colombia es un país bastante activo y siempre se destaca como una de las naciones con mayor actividad en lo referente a la apertura de empresas, lo cual, se puede considerar como un aspecto positivo. Sin embargo, profundizando un poco más en el tema, al indagar sobre las motivaciones que llevan a un colombiano a la exploración de una oportunidad de negocio, empiezan a surgir varios interrogantes. Aunque el Gobierno Nacional se encuentra implementando a partir del 2009 una Política nacional de Emprendimiento en el territorio colombiano, pareciera que ésta ha conllevado más a la consolidación de empresas y no a la apertura de nuevas organizaciones, el cual, debería ser el principal objetivo de dicho plan. Todo esto sumado a la inequidad en la distribución de la riqueza que ha perdurado por mucho tiempo en la sociedad colombiana. Por lo tanto, todo esto lleva a preguntarnos, si el origen de la pymes en Colombia es consecuencia de una acertada política nacional de emprendimiento o fruto de la ausencia de oportunidades laborales para los colombianos.

Al examinar las razones que motivan a un colombiano para la apertura de una empresa, queda demostrado que la "necesidad" sigue siendo un factor determinante, especialmente en el sexo femenino, el cual, debido a la constante discriminación en el mercado laboral, debe acudir al emprendimiento para poder subsistir. Aunque para los hombres la "oportunidad" sigue siendo su principal motivación, la necesidad sigue siendo un factor determinante en el momento de iniciar un nuevo proyecto laboral. Todo esto nos lleva a concluir que la falta de oportunidades laborales para los colombianos sigue siendo bastante alto, donde las mujeres y los jóvenes llevan la peor parte, y donde el acceso 
y remuneración de la mujer en el mercado laboral, debe ser definitivamente un tema urgente a revisar. Otro tema preocupante es la informalidad laboral, ya que está demostrado que la mayoría de apertura de empresas en Colombia se realizan en el mercado informal. Aunque en los últimos años el Gobierno Nacional ha intentado combatir este fenómeno, especialmente reduciendo la carga prestacional para las empresas con el fin de incrementar el empleo formal, la industria privada sigue siendo bastante mezquina frente a éste tema. La informalidad también se ha incrementado en gran medida debido a la falta de capital humano calificado, por lo que se debe reflexionar sobre éste tema y el rol que están cumpliendo las instituciones educativas, especialmente las enfocadas a tecnólogos y técnicos.

\section{REFERENCIAS}

Banco de la República, Documentos de Trabajo sobre Economía Regional, Informalidad laboral en las áreas urbanas de Colombia, Luis Armando Galvis, Cartagena de Indias, (Febrero de 2012).

Diario Portafolio, Sección Economía, Artículo "Informalidad laboral en Colombia llega al 51,3\%", (Febrero 6 de 2012).

Global Entrepreneurship Monitor (GEM), encuesta realizada a la población adulta (2007).

Ministerio de Industria, Comercio y Turismo, página web,
(2013), https://www.mincomercio.gov.co/

Ministerio de Industria, Comercio y Turismo, República de Colombia, Julio de 2009, Política Nacional de Emprendimiento de Colombia, versión final.

Ortega C. Algunos aspectos jurídicos para la conformación de empresas en Colombia. Revista de Investigación Semillas, Volumen 10, Enero - Diciembre, (2008).

Programa de las Naciones Unidas para el Desarrollo (PNUD), 29 de junio de 2012, "Informe sobre Desarrollo Humano 2011 - Sostenibilidad y equidad: Un mejor futuro para todos" (en español).

Portal razonpublica.com, artículo "La discriminación laboral tiene cara de mujer", (Mayo 4 de 2010).

Revista Dinero, Octubre 26 de 2012, Artículo "Pyme: sinónimo de oportunidad y desafío", Especial Comercial.

Superintendencia de Sociedades - Super-sociedades, página web, (2013), https:// www.supersociedades.gov.co/ 